Archivum, LXV, 2015, pp. 193-226

\title{
El diminutivo intensivo
}

Recibido: 22/03/2015

Aceptado: 31/08/2015

\section{RESUMEN:}

El objetivo de este artículo es, en primer lugar, describir el comportamiento semántico del diminutivo intensivo; en segundo lugar, deslindar dos modos de significación vehiculados por este sufijo. Para ello, adaptaremos a nuestro tema de estudio una teoría eminentemente semántica elaborada por Ducrot (1995), que tomaremos como punto de partida, para luego conducir nuestro análisis hacia otros horizontes teóricos, que nos permitirán dar cuenta, de manera precisa, de la distinción entre un sufijo intensivo de grado y un sufijo intensivo aspectual.

PALABRAS CLAVE: diminutivo, intensivo, sufijo de grado, sufijo aspectual.

\begin{abstract}
:
The aim of this paper is, first, to describe the semantic behavior of the diminutive intensive one; secondly, to define two manners of meaning carried for this suffix. For it, we will adapt to our topic of study an eminently semantic theory elaborated by Ducrot (1995), which we will take as a starting point, then to lead our analysis towards other theoretical horizons, which will allow us to realize, in a precise way, of the distinction between an intensive suffix of degree and an intensive aspectual suffix.
\end{abstract}

KEY WORDS: diminutive, intensive, suffix of degree, aspectual suffix.

\section{Introducción}

Este breve estudio pretende afinar la descripción semántica del valor intensivo atribuible a ciertos casos del sufijo diminu- 
tivo. Normalmente, ${ }^{1}$ este valor puede aparecer cuando el sufijo está ligado a bases adjetivales, participiales o adverbiales. Nosotros nos consagraremos al estudio de los dos primeros casos porque en ellos encontramos una sutil diferencia semántica en el modo de intensificación: un primer modo en donde el sufijo diminutivo funciona como un sufijo intensivo de grado, es decir, denota un alto grado de realización o de 'exceso' de la noción denotada por la base, y un segundo, en donde el sufijo diminutivo ligado a una subclase de adjetivos, los adjetivos perfectivoresultativos, y a participios de verbos de realización funciona como un sufijo intensivo aspectual que subraya el estado 'perfectamente' alcanzado denotado por el adjetivo o el participio.

Naturalmente, antes de hablar del matiz intensivo del sufijo, tendremos que presentar el fenómeno en toda su extensión, empezando por la definición de la noción de 'diminutivo'. Luego analizaremos los presupuestos que ella conlleva, considerándola a través del prisma de los posibles modos de atribución de la significación. Más adelante confrontaremos los valores intensivo y minorativo del sufijo valiéndonos de las nociones de 'realización' y 'desrealización' introducidas por Ducrot (1995). Esta perspectiva teórica va a permitirnos encauzar la descripción del diminutivo intensivo, pero pronto se revelará insuficiente en el análisis de ciertos casos. Necesitaremos, pues, encontrar otro modelo teórico para proponer una descripción exhaustiva que pueda dar cuenta de la diferencia que postulamos en este artículo entre los dos modos de denotación del diminutivo.

Dada la abundancia de sufijos diminutivos que posee la lengua española, nosotros escogeremos arbitrariamente el sufijo -ito, seguramente el más expandido de todos, en el cual es más fácil, para cualquier hablante de nuestra lengua, percibir las variaciones de sentido.

1 El sentido intensivo del sufijo podría aparecer también con sustantivos, pero solo en contextos exclamativos del tipo de: ¡Qué casita! Para estos casos, cf. García Negroni (1995). 


\section{Definición}

Si nos atenemos a la definición que el Diccionario de la lengua española $(D R A E)$ de la Real Academia Española da sobre la palabra diminutivo, vemos que, a través de las sucesivas ediciones, la primera acepción que encontramos en lo que concierne estrictamente al sufijo es: “Que denota disminución de tamaño en el objeto designado" ${ }^{2}$. Dejaremos de lado en este artículo el aspecto emotivo o apelativo, también citado en el DRAE a partir de la edición del Diccionario manual de 1983, II, en donde se especifica la ausencia de aminoración para esos casos. Lo que sí nos parece importante destacar aquí es la modificación incorporada a partir de la edición de 2001, en donde se menciona el aspecto intensivo del sufijo diminutivo ligado a ciertas bases adverbiales o adjetivales. Podríamos preguntarnos a qué obedece la tardanza del $D R A E$ en añadir este último aspecto que, sin embargo, resulta absolutamente evidente para cualquier hablante de la lengua española.

Pensamos que la respuesta a esta pregunta reside, no solamente en el peso de la tradición gramatical, sino, sobre todo, en el difícil equilibrio epistémico entre 'referencia', 'denotación' y 'sentido'.

\section{Referencia, denotación y sentido}

No abundaremos en todas las polémicas que estos términos han suscitado según los puntos de vista teóricos adoptados. En lo que respecta a nuestro trabajo, hemos elegido las ya clásicas definiciones de Lyons (1997) que se adaptan perfectamente a la demostración que pretendemos hacer en nuestro análisis. Según Lyons (1997: 107):

La diferencia crucial entre la referencia y la denotación es que la denotación de una expresión es invariante e independiente del

2 Cita de la edición electrónica de 2001. El artículo no fue enmendado para la 23ํㅡㄹ edición de 2014. 
enunciado; es la parte del significado que la expresión tiene en el sistema de la lengua, independientemente de su uso en ocasiones de enunciados particulares. La referencia, por el contrario, es variable y dependiente del enunciado (...). El sentido de una expresión es, simplemente, el conjunto o red de relaciones de sentido que se mantienen entre dicha expresión y otra cualquiera de la misma lengua.

Ahora bien, en el caso de la morfología derivativa, que es lo que nos ocupa aquí, el sentido de la expresión debería calcularse a partir del sentido de cada morfema que entra en la estructura de la palabra. De alguna manera seguimos el camino trazado por Lyons (1968: 196) cuando señala que una gran parte de la morfología derivativa "puede ser y debe ser integrada en las reglas de la sintaxis".

Veamos, de manera práctica, cómo entendemos el funcionamiento de estos tres conceptos.

Supongamos que alguien profiere el enunciado siguiente:

(1) Tiene un piso grande.

Supongamos también que este enunciado 'refiere' a un piso de $150 \mathrm{~m}^{2}$. Comparemos ahora con el siguiente:

(2) Tiene un piso grandecito.

Postulemos que este enunciado hace referencia a un piso más pequeño, fiándonos en la denotación del sufijo, que podríamos parafrasear con el sintagma más pequeño que.

Consideremos ahora los siguientes enunciados: 
(3) Tiene un piso pequeño.

(4) Tiene un piso pequeñito.

Si (3) 'refiriera', por ejemplo, a un piso de $40 \mathrm{~m}^{2}$, forzosamente (4) haría referencia a un piso más pequeño. La denotación del sufijo seguiría siendo la misma. Sin embargo, si hacemos jugar la relación entre los morfemas (el morfema de base y el sufijo), obtendremos como resultado el 'sentido' del derivado en los enunciados (2) y (4). En el caso de (2) observamos una 'aminoración' del sentido del morfema de base, mientras que en (4) notamos una 'intensificación' del mismo.

\section{Modificadores de 'realización' y de 'desrealización'}

En los ejemplos citados podemos ver, y esto de manera intuitiva, que en el caso de la aminoración, existe una oposición semántica entre la denotación del morfema de base y la del sufijo diminutivo. En el caso del enunciado (1), el morfema de base es un adjetivo que modifica un sustantivo que refiere a un objeto del mundo, del cual se predica que 'supera en tamaño' a la mayoría de los objetos de su especie, de allí el efecto de atenuación que obtenemos con la derivación diminutiva en (2). Todo lo contrario ocurre con el antónimo del adjetivo en (3), ya que este predica del mismo tipo de objeto que tiene 'menor tamaño' que la mayoría de los objetos de su especie. El sufijo diminutivo aplicado al adjetivo indica un grado aún superior en la 'reducción del tamaño'.

Esta descripción intuitiva puede formalizarse en términos más precisos y es exactamente lo que hicieron los máximos representantes de la teoría de la argumentación en la lengua (TAL), Anscombre y Ducrot, cuando introdujeron los conceptos de 'coorientación' y de 'contraorientación'. Estos conceptos serán esenciales a la hora de definir lo que Ducrot (1995) llama modificadores de 'realización' (MR) y de 'desrealización' (MD) (modificateurs réalisants et déréalisants). En principio, estos modifi- 
cadores, adjetivos o adverbios, se aplican a sustantivos o verbos. Ducrot los llamará, por comodidad, 'predicados'.

Ducrot (1995: 147) define estos modificadores de la siguiente manera:

Una palabra Y constituye un MD en relación con un predicado $\mathrm{X}$ si y solo si el sintagma XY:

a) No es considerado como contradictorio.

b) Tiene una orientación argumentativa inversa o una fuerza argumentativa inferior a la de $\mathrm{X}$.

Si XY tiene una fuerza argumentativa superior a la de $X, y$ la misma orientación, $\mathrm{Y}$ es un MR.

Para descubrir los MD, Ducrot propone el criterio siguiente: siendo $\mathrm{Y}$ un $\mathrm{MD}$ en relación con $\mathrm{X}$, se debe poder enunciar ' $X$, pero $X Y$ ' "sin tener una razón argumentativa precisa para oponer X a XY". Así, Ducrot nos da el ejemplo del adjetivo lejano considerado como un MD en relación con el sustantivo pariente:

(5) Pedro es un pariente, pero (un pariente) lejano.

En cuanto a los MR, Ducrot utiliza como criterio la posibilidad de inserción del conector incluso en una frase del tipo de: ' $X$, e incluso $X Y^{\prime}$. Para continuar con el ejemplo relacionado con el sustantivo pariente, Ducrot considera que el adjetivo cercano es un MR de pariente:

(6) Pedro es un pariente, e incluso (un pariente) cercano. 
Si invirtiéramos los adjetivos, obtendríamos, según Ducrot, unos enunciados que demandarían un esfuerzo interpretativo, algo completamente innecesario en los ejemplos (5) y (6). Ducrot los marca con el signo (\#) para indicar que su rareza no indica necesariamente agramaticalidad.

(7) \#3Pedro es un pariente, pero (un pariente) cercano.

(8) \#Pedro es un pariente, e incluso (un pariente) lejano.

No tenemos que perder de vista que estas definiciones se aplican a relaciones semánticas perceptibles en la combinación de estos modificadores con sus 'predicados' (sustantivos o verbos). Nosotros las adaptaremos al campo de la morfología derivativa. De todas maneras, Ducrot (1995: 153) no excluye la posibilidad de aplicar estas reglas al campo de los morfemas: “(...) una generalización es posible, que permitiría tratar morfemas como poco y un poco", considerados como MD en sus dos variantes, el primero sería un 'inversor' de la orientación argumentativa del sintagma en el que aparece; el segundo, un 'atenuador', que conservaría la orientación argumentativa, atenuando su aplicación en pos de una determinada conclusión. En el apartado siguiente retomaremos esta idea de 'atenuación', pero esta vez relacionada con el sufijo diminutivo.

\section{Estructura semántica del derivado}

En conversaciones mantenidas con el propio Ducrot en el marco de los seminarios que él impartía en la École des hautes études en sciences sociales de París, este nos sugirió un tratamiento posible para el diminutivo español, inspirado directamente en

3 Habría que aclarar que el signo (\#) es necesario solo cuando el enunciado no está acompañado de una prosodia enfática sobre el modificador cercano. Como se indica en García Negroni (op. cit.), dicha prosodia hace del modificador un modificador 'sobrerrealizante' y el enunciado resulta totalmente comprensible. 
su teoría de modificadores de 'realización' y 'desrealización'. La idea de Ducrot era que el sufijo diminutivo ligado a un adjetivo o adverbio "que iba en el sentido de la mayor cantidad (dans le sens de la grandeur)" operaba como un MD atenuador. Había, en este caso, 'contraorientación' entre el sentido del sufijo y el del morfema de base. Al contrario, si el sufijo estaba ligado a un adjetivo o adverbio "que iba en el sentido de la menor cantidad (dans le sens de la petitesse)", estábamos ante un caso de 'coorientación'. El sufijo actuaba, pues, como un reforzador de la base, generando el sentido 'intensivo', para utilizar el metalenguaje que nosotros emplearemos en este artículo.

Esta pista, que parece no contradecir la intuición de ningún hablante nativo de la lengua española, constituye un primer paso para el análisis del fenómeno. Lo que tenemos que tener en cuenta es este aspecto de microestructura que tiene el análisis morfológico, al cual estamos aplicando reglas que se verifican en un nivel de estructura superior, en este caso, las reglas de los MD o MR respecto a lo que Ducrot llama 'predicado'. Verificamos aquí, mutatis mutandis, la cita de Lyons sobre el hecho de que una gran parte de la morfología derivativa "puede ser y debe ser integrada en las reglas de la sintaxis"; en nuestro caso, las reglas semánticas relacionadas con la estructura sintagmática pueden aplicarse también a la estructura morfológica.

Si consideramos en un primer momento la estructura mínima, es decir, el juego entre los morfemas que componen una palabra, veremos que un adjetivo como grande puede ser considerado, en muchos casos, como un MR en relación con un sustantivo determinado, como en nuestro ejemplo, aplicado a piso. Si aplicamos a este adjetivo el sufijo diminutivo, obtendremos la estructura siguiente: [MR[MD]]. Vemos de esta manera, muy claramente, el fenómeno de 'contraorientación' de los morfemas. Habría que decir que en esta microestructura, el sufijo funciona como un MD de atenuación con respecto a la base. El derivado debería ser considerado como un MR (atenuado) en relación con el sustantivo piso. De la misma manera, el adjetivo pequeño, pue- 
de ser considerado en muchos casos como un MD, sobre todo aplicado a piso, al añadir el sufijo diminutivo, obtendríamos la siguiente estructura: [MD[MD]], vemos aquí un fenómeno de 'coorientación', lo que indicaría que el sufijo tendría que comportarse como un MR, en relación con el sentido del morfema de base. De esta manera, el derivado pequeñito se convertiría en un MD con mayor poder de 'desrealización' que el primitivo respecto al sustantivo piso.

La confirmación lingüística de esta descripción la podemos ver en la paráfrasis que sirve para describir el sentido de cada uno de los casos. En el primero, es decir, el del MR (atenuado), esta se realizaría con la ayuda del adverbio bastante, mientras que en el segundo, utilizaríamos el adverbio muy. Un piso grandecito se interpretaría como un piso bastante grande, contrariamente a un piso pequeñito, interpretable como un piso muy pequeño. Es por eso que, teniendo en cuenta el grado superlativo del adverbio utilizado en esta última paráfrasis, quizá sea más sensato hablar de 'intensificación' y no de 'refuerzo'.

\section{Estructura del derivado en el enunciado}

Acabamos de ver que, en un caso, el derivado se presenta como un MR (atenuado) en relación con el sustantivo piso. Veamos el funcionamiento de este primer caso dentro del enunciado. Supongamos, por ejemplo, el contexto siguiente: un diálogo entre dos amigos, uno de ellos planea un viaje a Barcelona y no sabe si podrá alojarse en casa de otro amigo porque no está al corriente de si ya ha alquilado un apartamento o si todavía reside en casa de sus padres. El que escucha podría responderle: -Pienso que te va a poder recibir, y luego encadenaría con:

(9) Tiene un piso, e incluso (yo diría) un piso grande.

(10) Tiene un piso, e incluso (yo diría) un piso grandecito. 
(11) ? Tiene un piso grandecito, e incluso (yo diría) un piso grande.

En el segundo caso que hemos expuesto, el derivado aparece como un MD con un grado superior de 'desrealización' que el primitivo en relación con el sustantivo piso. Para continuar con el ejemplo, habría que añadir una negación a la frase del amigo que aconseja: -Pienso que no te va a poder recibir.

(12) Tiene un piso, pero pequeño.

(13) Tiene un piso, pero pequeñito.

(14) Tiene un piso pequeño, e incluso (yo diría) un piso pequeñito.

Los encadenamientos (11) y (14) revelarían este juego de fuerza argumentativa. Según el análisis tradicional de la TAL, en un encadenamiento argumentativo, el conector incluso ${ }^{4}$, introduce un argumento más fuerte relacionado con el primer argumento del encadenamiento en pos de una determinada conclusión. Es verdad que la lengua española posee otras astucias para marcar el alto grado, quizá más naturales que el conector incluso, que en muchos casos necesita un refuerzo a modo de parentética, como lo hemos indicado en los ejemplos, para ser aceptado en este tipo de encadenamiento enunciativo. Pero el reparo que hemos introducido en la aceptabilidad de (11) se debe no tanto al conector, sino al sentido mismo del adjetivo derivado, que puede no ser interpretado inmediatamente como un minorativo de grande.

Este fenómeno de ambigüedad que constatamos en esta forma diminutiva parece ser una característica de los adjetivos de dimensión que expresan 'mayor cantidad' como larguito, anchito,

4 Trad. del conector même de la lengua francesa. 
altito. El primer escollo lo encontramos en el uso, tenemos que reconocer que son formas mucho menos utilizadas que sus antónimos: cortito, estrechito, bajito, cuyo sentido intensivo no deja duda. En los casos de larguito, anchito y altito, así como en el caso anteriormente expuesto del derivado de grande, deberíamos encontrarnos, según la regla, con el esquema siguiente en lo que se refiere al componente morfológico de los derivados: [MR[MD]]. De esta manera, el derivado, en relación con un predicado determinado, tendría que comportarse como un MR (atenuado), es decir, con una fuerza argumentativa inferior a la del adjetivo en su grado positivo. Sin embargo, en algunos de los ejemplos que veremos a continuación la adecuación a la regla no parece tan evidente:

(15) A: - ¡Ah! Por ahí en una hora, le... le puedo llamar en una hora larguita.

B: - Llámeme en dos horas, yo espero ${ }^{5}$.

(16) P. En YouTube abundan los vídeos de sus mejores puntos. ¿También se emociona con ellos?

R. Hubo uno que me dio energía (...). Era larguito. Duraba 14 minutos $^{6}$.

(17) Partía de muy poco pecho, (...) más bien era todo espalda porque soy anchita y me pusieron [una prótesis de] $455 \mathrm{~cm}^{3}$ submuscular?.

5 REAL ACADEMIA ESPAÑOLA: Banco de datos (CREA) [en línea]. Corpus de referencia del español actual. <http://www.rae.es> [29/06/2015].

6 El País, 09/06/2014: DEPORTES [Entrevista a Rafael Nadal].

7 http://foro.enfemenino.com/forum/chirurgie/_f90577_chirurgie-Doble-surco.html. [Las prótesis van de 100 a $500 \mathrm{~cm}^{3}$ ]. 
(18) Córteme la blusa anchita de sisa, porque muy al cuerpo me molesta ${ }^{8}$.

(19) Se lo dices desde aquí, altito, para que te oigan?

(20) ¡Niño, qué altito estás! Y lo que vas a crecer todavía ${ }^{10}$.

Los diminutivos, en los ejemplos impares de esta última serie, parecen tener un sentido intensivo, contrariamente a los de los enunciados pares en donde el sufijo tiende a una lectura minorativa ${ }^{11}$.

Para confirmar esta idea intuitiva, veremos si efectivamente el adjetivo diminutivo posee un valor de MR o de MR (atenuado) respecto al 'predicado' (el sustantivo o el verbo, según la terminología de Ducrot).

8 Ejemplo inspirado en un caso citado en el corpus CREA: Ten cuidado, no me lo hagas estrecho de sisa que luego hago el ridículo en las Cortes cuando presento las mociones de censura. Digo: ¡eso no está bien! y se me rompe por aquí, o sea que hazlo anchito de la sisa. Cf. REAL ACADEMIA ESPAÑOLA: Banco de datos (CREA) [en línea]. Corpus de referencia del español actual. <http://www.rae.es> [29/06/2015]. Un caso similar se encuentra en el Corpus del Español: En estas cavilaciones pasó algunos días, y como se decidiese a hacerse nueva levita y no quisiese dar su brazo a torcer al sastre, encargó a éste que se la hiciese bien anchita para poderse poner ropa debajo así que viniese el frío (...). Cf. Davies, Mark. (2002-) Corpus del Español: 100 million words, 1200s-1900s. Available online at http://www.corpus delespanol.org [29/06/2015].

9 REAL ACADEMIA ESPAÑOLA: Banco de datos (CREA) [en línea]. Corpus de referencia del español actual. <http://www.rae.es> [29/06/2015].

10 Ejemplo inspirado en un caso citado en el corpus CREA: -jAy, cómo está, figúrate, el niño ya por Dios -profirió la Villacantero, acaparando toda la atención-, dame un beso, un beso, guapo, figúrate, Mercedes, qué nieto de locura tienes ya, precioso, dame un beso, a ver, qué altito estás, figúrate...! Cf. REAL ACADEMIA ESPAÑOLA: Banco de datos (CREA) [en línea]. Corpus de referencia del español actual. $<\mathrm{http}: / / \mathrm{www} . \mathrm{rae}$. es> [29/06/2015].

11 Las dudas de los hablantes persisten al respecto. 
En el ejemplo (15), el adjetivo diminutivo funciona aparentemente como un MR en relación con el sustantivo hora. En este caso el adjetivo diminutivo no difiere prácticamente del significado del positivo, podríamos así reemplazar larga por larguita sin alterar el sentido de 'realización'. En todo caso, no podríamos establecer una diferencia en la fuerza argumentativa de ambas formas, es por eso que la secuencia siguiente parece extraña:

(21) ?? Una hora larguita, e incluso (yo diría directamente) larga.

Los encadenamientos posibles para el sintagma una hora larguita serían:

(22) Una hora larguita, incluso (hora y media / dos).

Así parece haberlo entendido el individuo B del diálogo presentado en (15).

En cuanto al ejemplo (16), el adjetivo diminutivo, en relación con el sustantivo vídeo, tendría el valor de un MR (atenuado). En este caso el reemplazo por el positivo suprimiría la atenuación, vehiculada por la forma diminutiva:

(23) Un vídeo larguito, e incluso (yo diría directamente) largo.

(24) ?? Un vídeo largo, e incluso (yo diría directamente) larguito.

Un caso similar al del ejemplo (15) aparece en el ejemplo (17). No habría una gran alteración de significado al conmutar 
el adjetivo en su grado positivo por el adjetivo diminutivo. Aquí tampoco podríamos distinguir claramente una diferencia en la fuerza argumentativa entre el adjetivo en su grado positivo y la forma diminutiva:

(25) ?? Soy anchita, e incluso (yo diría directamente) ancha.

Habría que añadir en el análisis de este caso el problema de la lectura eufemística impuesta por la forma diminutiva cuando esta es empleada para denotar cualidades axiológicamente marcadas hacia lo negativo, como veremos más adelante al analizar el caso de gordito.

Sin embargo, si consideramos el valor del diminutivo anchita en el ejemplo (18), vemos que posee un matiz atenuador con respecto al positivo:

(26) Me cortó la blusa anchita de sisa, e incluso (yo diría directamente) ancha.

Otra equivalencia en el sentido del adjetivo diminutivo y del adjetivo en su grado positivo con respecto al predicado la encontramos en el ejemplo (19):

(27) ?? Se lo dices desde aquí, altito, e incluso (yo diría directamente) alto, para que te oigan.

En el último ejemplo de nuestra serie, el ejemplo (20), el valor del adjetivo diminutivo depende de alguna manera de la denotación del sustantivo niño. La idea de atenuación se calcula a partir 
del significado del sustantivo. Altito funcionaría como la atenuación de alto, si admitimos que cuando hablamos de las características físicas asociadas a un niño, así como cuando nos referimos a las partes del cuerpo del mismo, muy a menudo empleamos un diminutivo con función de atenuador: la manito del niño, sus ojitos, su boquita, etc. Por ello, establecer alguna diferencia en la fuerza argumentativa del adjetivo diminutivo con respecto al positivo no parece natural:

(28) ? ¡Niño, qué altito estás, e incluso (yo diría directamente) qué alto estás! Y lo que vas a crecer todavía.

Podríamos insertar entre estos ejemplos el caso del diminutivo gordito, opuesto a delgadito, este último, según lo previsto en la regla, de matiz intensivo. El diminutivo gordito debería tener la misma ambigüedad de sentido que los diminutivos de adjetivos de dimensión que expresan 'mayor cantidad', pero la norma en este caso parece ser privilegiar el sentido minorativo:

(29) La imagen de Cupido, el niño gordito con alas.

(30) ? La imagen de Cupido, el niño gordo con alas.

Para explicar la mayor naturalidad del ejemplo (29) con respecto al (30), habría que repetir dos razones ya evocadas en el análisis del ejemplo (20): la preferencia de la forma diminutiva impuesta por el significado del sustantivo niño y el hecho de que el español prefiera la forma diminutiva para atenuar características axiológicamente marcadas hacia lo negativo. Esta última característica, que nosotros hemos llamado 'lectura eufemística', hace que el recurso a una explicación de tipo 'referencialista' resulte superfluo: las formas gordo /-a, gordito / -ita podrían predicarse de un mismo individuo sin referencia a una supuesta 
diferencia en la escala del peso de la persona. Por ejemplo, a la pregunta: ¿Quién es Berta?, podríamos responder:

(31) Es aquella chica gordita.

Si reemplazáramos gordita por gorda, obtendríamos una frase 'políticamente incorrecta'. Si se tratara de una frase insultante, sería muy difícil cambiar el positivo por el diminutivo:

(32) Pero ¿quién se habrá creído la gorda esta?

La razón de la preferencia del positivo no obedece tanto al sentido minorativo que podríamos ver en el diminutivo, sino más bien a la falta de adecuación del sentido eufemístico en este tipo de contexto.

La tentación de ampliar este análisis a los adverbios de lugar con sufijo diminutivo lejitos y cerquita sería una consecuencia lógica dentro de este marco teórico; sin embargo, las atestaciones que hemos encontrado de la forma lejitos no permiten percibir la ambigüedad de sentido que acabamos de ver en los adjetivos que expresan 'mayor cantidad'. En lo que concierne a cerquita, el sentido es intensivo, concordando con la regla de los adjetivos que expresan 'menor cantidad'.

Veamos estos ejemplos en donde el sentido minorativo del sufijo no se ve claramente:

(33) Es difícil llegar a esa playa: está lejitos.

(34) *Es fácil llegar a esa playa: está lejitos.

(35) *Es fácil llegar a esa playa: está lejitos, pero no lejos. 


\section{Condicionamiento contextual}

A la luz de lo analizado en los apartados anteriores, podemos rendirnos a la evidencia de que la regla enunciada por Ducrot tiene una aplicación aleatoria en los contextos relacionados con los adjetivos diminutivos que constituyen nuestro objeto de estudio. La dependencia contextual del significado se vislumbra, pues, como un criterio posible para obtener una descripción más precisa del fenómeno que estamos estudiando.

La teoría que hemos presentado en los apartados anteriores implica necesariamente una concepción gradual del análisis semántico. Por ello, los ejemplos que se adecuan fácilmente a este tipo de análisis son aquellos en los cuales existe un tipo preciso de relación de antonimia: los antónimos de grado. En cuanto nos apartamos de los casos 'medibles' con precisión en una determinada escala, la interpretación del valor del diminutivo parece complicarse. En efecto, no todos los antónimos de grado pueden graduarse en una escala numérica:

(36) La crema (está / ha quedado / ha salido) (durita / blandita).

Tenemos aquí un adjetivo que va en el sentido de la 'mayor cantidad de algo' en este caso preciso la 'mayor cantidad de dureza'. Según hemos visto, aquí el diminutivo debería funcionar como un atenuador respecto al sentido de la base. Sin embargo, si nos fijamos en el sentido del sustantivo modificado por la forma diminutiva, veremos que el sentido mismo de la palabra crema, en la acepción que nos interesa, presupone una sustancia líquida, espesa o densa, pero nunca dura. Decir que la crema ha salido durita podría equivaler aquí a un eufemismo. Esto se puede ver también en la paráfrasis con el adverbio bastante, cuya ambi- 
güedad ${ }^{12}$ no nos es de ningún auxilio en el momento de interpretar, aunque claramente aquí no queremos decir 'ni muy dura ni poco dura', sino 'notablemente dura':

(37) La crema (te) ha salido bastante durita.

La misma conclusión que podríamos sacar del adjetivo en su grado positivo valdría también para el diminutivo ${ }^{13}$ :

(38) La crema (está / ha quedado / ha salido) (dura / durita): está incomible.

Sin embargo, como ya lo hemos señalado para este tipo de casos (cf. ejemplos 18-20), siempre cabe la posibilidad de la interpretación atenuadora, aunque, de todas maneras, en el caso del ejemplo (39), relacionado con el sustantivo crema, el encadenamiento parezca menos natural que en el ejemplo (40):

(39) ? La crema te ha salido durita, pero no dura.

12 En el artículo enmendado de la vigesimotercera edición del DRAE (2014), podemos ver claramente esta ambigüedad si consideramos las acepciones tanto de la forma adjetival como adverbial:

1. adj. Que basta o es suficiente.

2. adj. Abundante, copioso. Recorrieron bastantes kilómetros. Cayó bastante agua.

3. adv. c. Ni mucho ni poco, ni más ni menos de lo regular, ordinario o preciso; sin sobra ni falta.

4. adv. c. En cantidad notable. Es bastante rico. Es bastante bella.

13 Cf. otro ejemplo del tipo: Mi panza se pone durita. Hola quiero saber por qué se me pone dura la panza. http://espanol.babycenter.com/thread/242691/mi-panza-sepone-durita- 
(40) (...) así que había que vidriarla cuando la pieza [de alfarería] estaba durita y no seca ${ }^{14}$.

Otro contexto en donde no se ve claramente el efecto atenuador del sufijo con respecto al adjetivo es el siguiente:

(41) El tipo apareció con el pelpa en la manopla. "Mirá, le dijo, antes que me digas nada, acá está mi divorcio" y acto seguido, se metió en la ducha. Ella quedó durita ${ }^{15}$.

Veamos otro ejemplo con un contexto más explícito. Se trata de un diálogo entre un hombre y una mujer, en una obra de teatro $^{16}$. La mujer se acerca al hombre y le toca, seguramente, la parte del brazo correspondiente al bíceps:

(42) Huuuummm, tenés durito (palpándole el brazo, admirativamente), se ve que tenés fuerza...

Es evidente que, de acuerdo con el sentido admirativo del enunciado, el sentido de durito no es aquí 'un poco duro' o 'bastante', en el sentido de 'ni mucho ni poco', sino 'muy duro'.

Podríamos realizar un análisis similar del adjetivo antónimo. Si volvemos al ejemplo inicial, el sintagma una crema blandita admite igualmente las interpretaciones intensiva y minorativa. Sería posible insertar en él tanto los adverbios intensivos bien 'pon-

14 REAL ACADEMIA ESPAÑOLA: Banco de datos (CREA) [en línea]. Corpus de referencia del español actual. <http://www.rae.es> [29/06/2015].

15 Ibídem [29/06/2015].

16 Ibídem [15/03/2015]. 
derativo' o muy 'superlativo' como el adverbio bastante en sus dos sentidos: 'atenuador' e 'intensivo'.

Sin embargo, en algunos casos el contexto podría condicionar una interpretación determinada. En el siguiente ejemplo la lectura intensiva parece primar:

(43) Le he servido todita la carnecita blandita, nadita de nervio le he puesto ${ }^{17}$.

El encadenamiento con la segunda parte del enunciado apoya esta interpretación. En efecto, sería más natural introducir como refuerzo los adverbios intensivos anteriormente citados. Si insertáramos el adverbio bastante, este se comprendería en su sentido intensivo.

El mismo análisis podríamos hacer en el próximo ejemplo:

(44) Cuando esté blandita la carne, se procede a deshilachar en hebras finas ${ }^{18}$.

El sentido atenuador puede recuperarse en el ejemplo que damos a continuación:

(45) (...) vaya agregando harina mientras bate con cuchara de madera hasta formar una masa blandita ${ }^{19}$.

17 Ibídem [29/06/2015].

18 Ibídem [29/06/2015]

19 Ibídem [29/06/2015]. 
Este último ejemplo admitiría un refuerzo con el adverbio bastante en su sentido atenuador. No habría un sentido contradictorio en el encadenamiento siguiente:

(46) Una masa bastante blandita, pero no blanda.

Contrariamente a los casos anteriores:

(47) ?? Le he servido todita la carnecita bastante blandita, pero no blanda, nadita de nervio le he puesto.

(48) ?? Cuando esté bastante blandita la carne, pero no blan$\mathrm{da}$, se procede a deshilachar en hebras finas.

Hay casos de antónimos de grado aún menos medibles que los precedentes en una supuesta escala. Por ejemplo:

(49) Un niño (contentito / tristecito).

(50) El niño está (contentito / tristecito).

(51) Un niño (buenito / malito).

(52) El niño es (buenito/malito).

¿Qué valor debemos atribuir al diminutivo en cada caso? Aquí no podemos utilizar los criterios de 'realización' o 'desrealización'. Sería absurdo sostener que, por ejemplo, los adjetivos contento y bueno 'realizan' el sustantivo niño o que sus antónimos lo 'desrealizan'. En los ejemplos que hemos encontrado de estos casos, la lectura intensiva parece imponerse. Hemos escogido los enunciados en los cuales aparece el adverbio bastante en su sentido intensivo. Pensamos que si es posible reforzar el 
sentido intensivo con el adverbio bastante, con mayor razón lo podremos hacer con los adverbios intensivos bien y muy.

(53) Se le notaba ayer bastante contentito e incluso risueño, sin duda porque los buenos resultados están sirviendo de medicina para los males pasajeros ${ }^{20}$.

(54) Obama bastante contentito porque tiene el "as" debajo de la manga (la posible captura del Güero Palma) ${ }^{21}$.

(55) Presiento que el capítulo que sigue va a ser muy triste (...). Voy a llorar seguramente (...), no sé porque presiento que va a ser bastante tristecito...22

(56) Bueno, de veras que no se cansa una de registrar la sarta de patinones que dan en la presidencia municipal de Zacatecas, y el más reciente resbalón estuvo relacionado con un tema bastante tristecito a nivel nacional. Luego de la explosión en un edificio de Pemex, del que se han dicho y especulado montones de teorías, el presidente Peña Nieto declaró eso que llaman "luto nacional" por tres días para honrar la memoria de casi 40 personas que fallecieron $a^{2} i^{23}$.

(57) Tengo un perro labrador que se llama Harry, es un poco hincha pero es bastante buenito ${ }^{24}$.

20 http://www.diariodecadiz.es/article/cadizcf/1491186/default.html

21 https://www.facebook.com/animalpolitico/posts/327363753981000

$22 \mathrm{http} / /$ www.gleeklatino.com/t18910p165-ficklaine-gracias-por-formar-partede-mi-epilogo-actualizado-13-06-2014

$23 \mathrm{http}: / /$ ntrzacatecas.com/2013/02/05/la-manguera-478/

24 http://www.potterfics.com/perfil/2131 
(58) Y es bastante ingenuo. Bastante buenito. Nada parecido con los chicos que usualmente me atraen. ¡Es demasiado buenito para mí! ${ }^{25}$

(59) DJ bastante malito igual que el grupo en vivo ${ }^{26}$.

(60) Bastante malito el paraguayo Enrique Cáceres, en una acción dos penales a favor de México que no se sancio$\operatorname{nan}^{27}$.

¿A qué se debe esta tendencia a la lectura intensiva? Posiblemente sea una interpretación aspectual derivada de aquellos casos en los que normativamente aparece esta lectura, como pequeñito, cortito, estrechito, bajito, delgadito. El sentido de estos diminutivos, como lo hemos visto, está menos sujeto a controversias que los antónimos correspondientes. Así, estas formas, con su correspondiente interpretación intensiva, funcionarían como el término marcado, de ahí la posibilidad de la extensión analógica del sentido intensivo.

\section{Consideraciones aspectuales}

Analizaremos aquí una clase de adjetivos y de participios. Los adjetivos pertenecen a la vasta clase de adjetivos episódicos, entre los cuales tenemos la subclase de adjetivos perfectivos. Según la definición de Bosque (1990: 178) los adjetivos perfectivos denotan "un estado que se interpreta como el resultado de una acción o un proceso", de ahí la relación semántica que poseen con los participios que denotan "resultados de acciones o procesos verbales que desembocan en un determinado estado". Tanto unos como otros pueden aparecer en estructuras atributivas (re-

25 http://cronicadeunasilueta.blogspot.fr/2009/12/la-llamada.html

26 https://es.foursquare.com/v/barezzito-condesa/54cea921498ea0f009077ba3

27 http://www.tagthebird.com/us/tweet/5590237 
gidas por estar copulativo) y predicativas, seleccionadas o adjuntas, orientadas hacia el sujeto o el objeto.

Veremos aquí algunos ejemplos, de predicativos seleccionados introducidos por verbos pseudocopulativos como quedar (ergativo), quedarse (inergativo o ergativo) o salir (en su versión pseudocopulativa con matiz resultativo). Confrontaremos en algunos casos los pseudocopulativos con el verbo estar en sus dos versiones: copulativa y pasiva ${ }^{28}$ :

(61) La niña (está / ha quedado) cansadita. [Atributo / predicativo seleccionado].

(62) El perro (está / se ha quedado) quietito. [Atributo / predicativo seleccionado].

(63) El abuelo (está / se ha quedado) dormidito. [Atributo / predicativo seleccionado].

(64) Elisa (está / ha quedado) desesperadita por el carácter del marido. [Pasiva de estado ${ }^{29}$ / predicativo seleccionado].

(65) Las rosquillas te han salido quemaditas. [Predicativo seleccionado].

En estos casos, el aspecto resultativo del adjetivo, ligado al aspecto resultativo del verbo, parece determinar una preferencia hacia una lectura intensiva del diminutivo. En los pocos ejemplos que hemos encontrado en el corpus $\mathrm{CREA}^{30}$, los diminutivos

28 En la frase copulativa con estar se asigna una propiedad extrínseca al sujeto, mientras que, en la frase pasiva, el estado es una consecuencia de una acción que afecta al sujeto.

29 Forma activa: El carácter de su marido desespera a Elisa.

30 REAL ACADEMIA ESPAÑOLA: Banco de datos (CREA) [en línea]. Corpus de referencia del español actual. <http://www.rae.es> [30/06/2015]. 
también tienen esta lectura. En algunos casos aparecen modificados por el intensivo muy. En uno de los ejemplos veremos un caso de reduplicación, figura que refuerza la denotación del alto grado:

(66) Con los dedos se estiró las bolsas de piel debajo de los ojos, se limpió los restos de lápiz y volvió a delinearse la ceja. ¿Está triste, cansadita la niña? Pensó que le gustaría volver a nacer $(. .$.$) .$

(67) Estás muy cansadita, ¿verdad, niña mía? Anda, túmbate aquí, que es como tumbarte en el útero donde te concebiste (...).

(68) Alianza Cívica considera que la ola democratizadora a la que se han subido la mayoría de la prensa, radio y televisión en el Distrito Federal no ha llegado (o lo hace "muy cansadita", según sus portavoces) al interior del país (...).

(69) Mildred. - (Cariñosa.) ¿Estás muy cansadito?

Williams. - (Gime.) Es terrible lo que una ninfomaníaca puede hacer con uno.

(70) Además debe dejársele quietito después de comer, ni siquiera cambiarle los pañales, y tratar de mantenerlo en brazos en posición vertical por un rato.

(71) Yo seguía las instrucciones y me quedaba quietito, quietito con cara de otario, como si no supiera nada.

(72) Otra gran pasión de los hijos es la cama. Cuando era pequeño podía estar horas y horas contemplándole dormidito en su cunita (...).

(73) - Ícaro, ¿trajiste el tocadiscos?

- ¿Mmm?

- Espérate, ¿no ves que está dormidito?

- Uuuy sí... a la ru ru nene, no mames. 
(74) (...) que la abuela, no te preocupes, siempre te quiso también y se murió dormidita, sin sufrir ni nada, como ella siempre quiso, de golpe y sin molestar a nadie.

(75) La Mary decía que ella se imaginaba a Jarabo, tan requeteguapo, quitándose la ropa y lavándose completamente en cueros, restregándose fuerte con una manopla y jabón Lagarto, desesperadito para no dejarse encima una gota de sangre $(\ldots)$.

(76) Los veraneantes-conductores que llegan desesperaditos y sudorosos a este bonito y fresco pueblo serrano (...).

(77) (...) nosotras tres pegando gritos como unas locas en el tribunal, desesperaditas (...).

(78) El quemadito hombrón, de 22 años, buceaba en un banco de coral frente a la playa Caves Beach, cerca de Newcastle, cuando fue atacado por un tiburón alfombra.

(79) Carlitos Alegre no cesaba de preguntar de qué playa lejana o de qué veraneo tropical llegaba tanta gente tan bronceada, tan uniformemente quemadita y morena (...).

(80) Es bueno guardársela [la mortaja desenterrada] quemadita y pulverizada (...).

En las frases regidas por verbos de 'realización'31, existe una estructura muy usual en español, que es la del 'participio cognado' con el verbo rector. En estas estructuras, el diminutivo posee claramente un valor intensivo de refuerzo de la denotación del estado final del proceso verbal. Demonte y Masullo (1999: 2492) consideran que los predicativos adjuntos orientados hacia el objeto, en donde aparecen estas formas cognadas, son una variante de los predicativos 'pseudoresultativos o de la manera del estado final', que ellos llaman predicativos de 'reiteración del 
estado final'. Como se trata de cuantificaciones del estado final, Demonte y Masullo (ibídem: 2493) hacen notar que en este tipo de estructura sería imposible introducir otros cuantificadores. Veamos su ejemplo:

(81) *Dobló mucho la ropa bien dobladita.

Vemos, sin embargo, que la presencia del adverbio aspectual bien es completamente compatible con este tipo de construcción, como lo señala Bosque (1990: 196): “(...) la construcción coloquial del participio cognado (...) con frecuencia requiere en español el adverbio aspectual bien".

Consideremos algunos ejemplos:

(82) Tostó el pan bien tostadito.

(83) Corta la cebolla bien cortadita.

(84) Pica la carne bien picadita.

(85) Cuece el bizcocho bien cocidito.

(86) Bate las claras bien batiditas.

(87) Seca las berenjenas bien (sequitas / *secaditas).

(88) Limpia el baño bien (limpito / *limpiadito).

En los ejemplos (87) y (88) vemos que la forma seleccionada es la forma adjetival y no la participial. Se trata, por supuesto, de adjetivos perfectivos que tienen un parentesco morfológico y semántico con el verbo rector, de tal manera que conforman un doble paradigma con el participio correspondiente al verbo. Se opera en estos casos una selección entre estas dos formas en posición predicativa, prefiriéndose el adjetivo perfectivo, espe- 
cializado en el resultado de la acción, al participio, relegado a la denotación de la acción acabada.

En esta última serie de ejemplos, vemos claramente que el sufijo diminutivo posee un valor intensivo puramente aspectual, opuesto al valor 'cuantificador' del sufijo, utilizado para ponderar el grado, tal como lo hemos visto en los ejemplos iniciales de este apartado. Las diferencias entre estos dos tipos de intensificación constituirán el objeto del apartado siguiente.

\section{El sufijo diminutivo intensivo: sufijo de grado o sufijo aspec- tual}

En este apartado abordaremos el problema del tipo de intensificación que puede denotar el diminutivo. Pensamos que el problema no puede circunscribirse solo al análisis semántico de los morfemas que componen el derivado, dado que, como lo hemos visto, este análisis no puede dar cuenta de un buen número de casos en donde el sufijo diminutivo funciona como intensivo. Habría que ampliar nuestra perspectiva analítica para explicar la sutileza semántica que podemos constatar en nuestros ejemplos. La clave reside en la sensible frontera existente entre el plano semántico y sintáctico, es decir, en las variaciones de sentido que producen ciertas combinaciones sintácticas.

Analizaremos entonces el comportamiento de los ejemplos que hemos visto en los apartados anteriores con los adverbios de grado bien, 'ponderativo' y muy 'superlativo', confrontados al adverbio bien ${ }_{2}$ 'aspectual' que refuerza el 'estado alcanzado' o el 'acabamiento' del proceso verbal. En los ejemplos que veremos a continuación, los dos valores del adverbio bien aparecerán en distribución complementaria. La selección entre estas formas es discriminante y nos permitirá separar el corpus de diminutivos intensivos en dos subclases. Por una cuestión de espacio, no abundaremos en ejemplos de casos en donde el adjetivo fun- 
ciona como especificativo en el sintagma nominal: un piso (bien / muy) pequeñito, un hombre (bien / muy) bajito, una niña (bien 1 muy) cansadita, un bizcocho (bien $/ \#^{32}$ muy) cocidito, etc. Preferimos reservar espacio para las frases atributivas o predicativas en donde podremos apreciar las variaciones de significado con mayor exactitud:

(89) El piso es (bien $/$ muy) pequeñito.

(90) Juan es (bien 1 / muy) bajito.

(91) La niña (está / ha quedado) (bien / muy) cansadita.

(92) El perro (está / se ha quedado) (bien 1 / muy) quietito. dita).

(93) La crema (está / ha quedado) (bien 1 / muy) (durita + blan-

(94) El abuelo (está / se ha quedado) (bien / muy) dormidito.

(95) Elisa (está / ha quedado) (bien 1 / muy) desesperadita por el carácter del marido.

(96) Las rosquillas te han salido (bien $/$ / muy) quemaditas.

(97) Tostó el pan (bien $/$ /\# muy) tostadito.

(98) Corta la cebolla (bien $/$ \# \#uy) cortadita.

(99) Pica la carne (bien $/$ / \#muy) picadita.

(100) Cuece el bizcocho (bien $/$ / \#muy) cocidito.

(101) Bate las claras (bien / \#muy) batiditas.

(102) Seca las berenjenas (bien $/$ / \#muy) sequitas.

(103) Limpia el baño (bien $/ 2$ \#muy) limpito.

32 El signo (\#) significa en estos ejemplos: incorrecto para reforzar el resultado de la acción perfectamente acabada. 
La conclusión que podemos sacar después de haber observado atentamente estos ejemplos es que el sufijo diminutivo puede tener un comportamiento divergente en cuanto al modo de intensificación. Vemos aquí un primer tipo de intensificación (del ejemplo 89 al 96), en donde el sufijo diminutivo intensivo se especializa en el grado superlativo. En estos casos el derivado diminutivo acepta de la misma manera los adverbios bien, y muy, estableciéndose entre ellos una semejanza en cuanto a la denotación del alto grado. Así, habría una equivalencia semántica ${ }^{33}$, por ejemplo, entre las secuencias un piso bien ${ }_{1}$ pequeñito y un piso muy pequeñito o entre una niña bien cansadita y una niña muy cansadita. A través de estos ejemplos vemos también que la naturaleza estable o episódica del adjetivo no altera en absoluto el sentido intensivo del sufijo.

En el segundo tipo de intensificación (del ejemplo 97 al $103^{34}$ ), el derivado diminutivo de naturaleza participial o adjetival, en posición predicativa, denota el aspecto 'perfectamente' acabado de la acción verbal. Su valor es, por consiguiente, aspectual o intensivo-aspectual para ser más exactos, ya que marca el grado máximo de realización del proceso verbal. En estos casos es posible reiterar ese grado máximo con el adverbio aspectual que hemos señalado.

En cuanto al matiz adverbial propiamente dicho, no podemos confundir el adverbio de grado bien ${ }_{1}$ con su homónimo aspectual. En el primer caso, como ya lo hemos indicado, el adverbio bien $_{1}$ es equivalente a muy. Al intercambiarlos en el sintagma, no

33 Cf. R.A.E. Diccionario panhispánico de dudas (2005), versión electrónica. En el artículo sobre el adverbio bien, se puede leer en el punto 2: Antepuesto a un adjetivo o a otro adverbio, funciona como intensificador enfático, con valor equivalente a muy: «Pues está bien claro» (Marsé Muchacha [Esp. 1978]); «Yo me vine a dormir porque era bien tarde» (Martín del Campo Carreteras [Méx. 1976]).

34 Casos del participio o del adjetivo perfectivo cognado. En estos casos, como ya lo hemos indicado, el predicativo reitera el significado del verbo de realización. 
obtenemos diferencias de sentido. En el segundo caso, este intercambio no es posible sin variación del sentido: el adverbio bien ${ }_{2}$ (aspectual) denota el límite máximo de 'completitud' del proceso verbal. El adverbio muy sobrepasa ese límite, eso es exactamente lo que hemos querido expresar con el símbolo \# delante del adverbio.

En la terminología de la tradición gramatical española contemporánea, el adverbio muy, en estos casos, debería ser considerado como un 'cuantificador de exceso'. Es lo que en una teoría puramente semántica, como la TAL, se llamaría un 'operador inversor'. El ejemplo clásico es el 'operador' demasiado 35 (Ducrot 1980, Anscombre et Ducrot 1983, Carel 1995). En los casos que nos ocupan, muy tendría un sentido muy cercano a demasiado. Un 'operador inversor' invierte la conclusión natural del enunciado en el que se inserta. El conector pero puede servirnos para expresar esta diferencia de matiz:

(104) Le he dicho una baguette bien cocida, pero no muy cocida.

\section{Conclusión}

Del análisis que hemos realizado en el presente artículo se desprende que el sentido del sufijo diminutivo intensivo se debe calcular no solo respecto a la estructura aspectual de la base a la cual se junta, sino también a la estructura aspectual del predicado. El sufijo diminutivo intensivo aspectual aparece ligado a bases adjetivales o participiales que denotan el estado perfectamente acabado de la acción verbal, regidas por verbos de realización. El sufijo diminutivo intensivo de grado no requiere una determinada estructura aspectual de la base ni tampoco un tipo específico de predicado. La relación semántica entre los dos tipos de sufijos intensivos funciona- 
ría entonces como una oposición privativa desde el punto de vista de la presencia o de la ausencia del rasgo aspectual 'perfectivo-resultativo' tanto en el morfema de base como en la estructura eventiva del predicado.

En la descripción semántica del diminutivo intensivo no sería superfluo introducir estos dos modos de significación que acabamos de analizar. Tendríamos, entonces, dos subclases de diminutivo intensivo: el intensivo de grado y el intensivo aspectual. Esta subdivisión no debe considerarse como una multiplicación innecesaria en la descripción, parafraseando el célebre principio de Ockham, sino como una necesidad para dar cuenta precisamente de una variación en el sentido de los enunciados.

\section{Referencias bibliográficas}

Anscombre, Jean-Claude: "La nature des topoï", en Théorie des topoï, Anscombre, J-C. (ed.), París, Éditions Kimé, 1995, págs. 49-84.

Anscombre, Jean-Claude: "Topique or not topique: formes topiques intrinsèques et formes topiques extrinsèques", Journal of Pragmatics, 24, 1995, págs. 115-141.

Anscombre, Jean-Claude: “Dénomination, sens et référence dans une théorie des stéréotypes nominaux", Cahiers de praxématique, 36, 2001, págs. 43-72.

Anscombre, Jean-Claude: "États et propriétés dans les combinaisons être + Adjectif", Le français moderne, 78, 2, 2010, págs. 186-205.

Anscombre, Jean-Claude: "El sufijo -ón en español contemporáneo: morfología y prototipos”, Oralia, 16, 2013, págs. 11-32.

Anscombre, Jean-Claude et Ducrot, Oswald: L'argumentation dans la langue, Bruselas, Pierre Mardaga, 1983. 
Bosque, Ignacio: "Sobre el aspecto en los adjetivos y en los participios", Tiempo y aspecto en español, I. Bosque (ed.), Madrid, Cátedra, 1990.

CAREL, Marion: "Trop: argumentation interne, argumentation externe et positivité", en Théorie des topoï, Anscombre, J-C. (ed.), París, Éditions Kimé, 1995, págs. 177-206.

Demonte, Violeta: Detrás de la palabra. Estudios de gramática del español, Madrid, Alianza Universidad, 1991.

Demonte, Violeta y Masullo, José Pascual: “La predicación: los complementos predicativos", Gramática Descriptiva de la Lengua Española, I. Bosque y V. Demonte (eds.), Colección Nebrija y Bello, Madrid, Espasa Calpe, 1999, págs. 2461-2523.

Ducrot, Oswald: “Les modificateurs déréalisants", Journal of Pragmatics, 24, 1995, págs. 145-165.

García Negroni, María Marta: "Scalarité et Réinterprétation: les Modificateurs Surréalisants", en Théorie des topoï, Anscombre, J-C. (ed.), París, Éditions Kimé, 1995, págs. 101-144.

Hernanz, M. Lluïsa: “En torno a la sintaxis y la semántica de los complementos predicativos en español", Estudi General, 8, 1988, págs. 7-27.

Lang, Mervyn F.: Formación de palabras en español. Morfología derivativa productiva en el léxico moderno. Título original: Spanish Word Formation (1990). Adaptación y traducción: Alberto Miranda Poza, Madrid, Cátedra, 1992.

Lyons, John: Introduction to Theoretical Linguistics, Cambridge, Cambridge University Press, 1968.

Lyons, John: Semántica lingüística. Una introducción. Título original: Linguistics semantics. An introduction (1995). Traducción y adaptación de Santiago Alcoba, Barcelona, Paidós, 1997.

Mattews, P. H.: Morfología. Introducción a la teoría de la estructura de la palabra. Título original: Morphology. An Introduction to 
the Theory of Word-structure. Traducido y adaptado por Rafael Monroy Casas, Madrid, Paraninfo, 1980.

Náñez Fernández, Emilio: El diminutivo. Historia y funciones en el español clásico y moderno, Madrid, Gredos, 1973.

Ángel Eduardo Toledo

ÉCOLE DES HaUtes ÉTUDES EN SCIENCES SOCIALES (PARÍS) 